\title{
Using NGS to answer biological questions
}

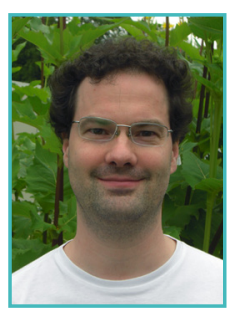

\section{Björn Usadel}

RWTH Aachen University and Forschungszentrum Jülich, Germany Usedal B (2014) EMBnet.journal 20(Suppl A), e771. http://dx.doi.org/10.14806/ej.20.A.771.

As generating next generation sequencing (NGS) lished these annotations, NGS data can then be data has become much cheaper, it is becoming more and more commonly used to address questions hitherto tackled by microarrays or by costly large scale EST sequencing. However, the computational challenge to analyse and interpret these data still remains.

Here we present some tools for the processing of NGS data. Focusing on RNA-seq data we show that using these tools, it is possible to get a first idea about major biological stories and to get a first overview which can then be used to develop biological hypotheses which can then be tested in more detail.

One example entails the often performed NGS analysis of non-model plant species and the exploration of metabolic pathways within these species (Lohse et al., 2014). Having estab-

analysed for statistical changes and explored for differences in expression which is demonstrated here (see Figure 1).

A main take home message however is that, even though these tools will help the experimenter in data analysis and interpretation, knowledge of the underlying biological system is of course required.

\section{Acknowledgements}

The authors would like to thank the BMBF for funding of the plant primary database 0315961.

\section{References}

Lohse M, Nagel A, Herter T, May P, Schroda M, Zrenner R, Tohge T, Fernie AR, Stitt M, Usadel B (2014) Mercator: A fast and simple web server for genome scale functional annotation of plant sequence data. Plant Cell Environ. 37(5):1250-8. http://dx.doi.org/1011111/pce.12231.

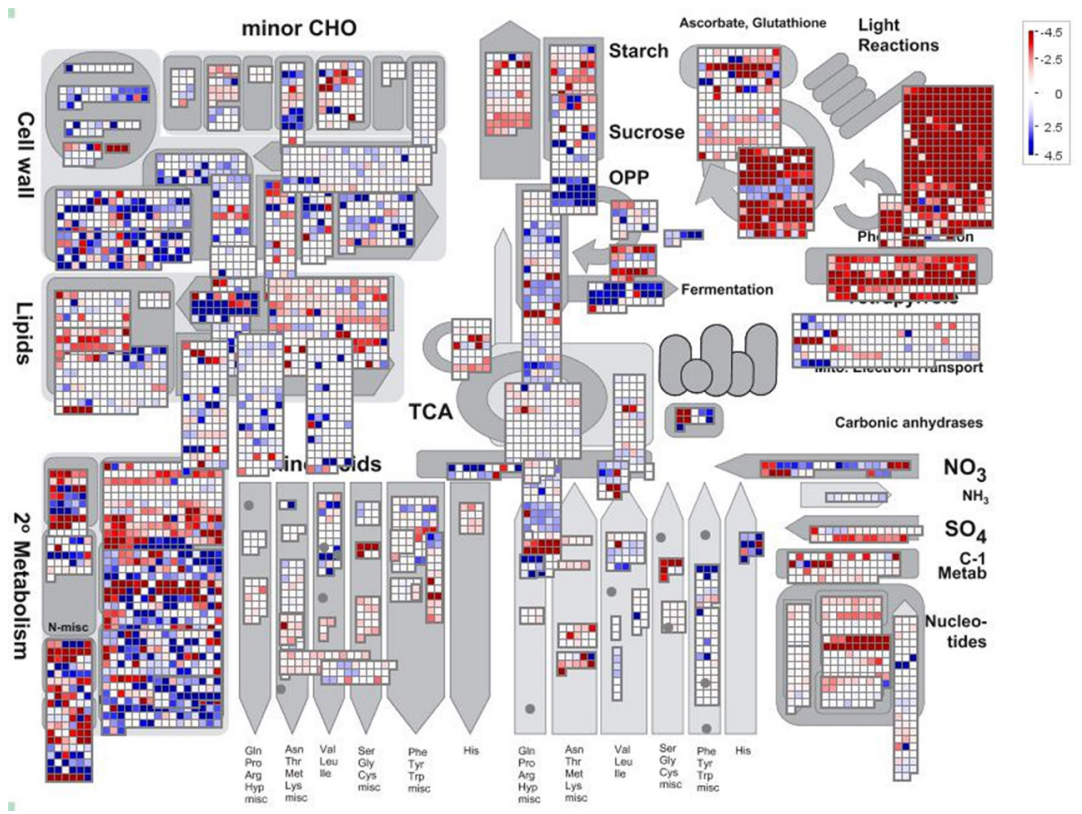

Figure 1. 